\title{
Expansion of Hypergeometric Functions in Series of Other Hypergeometric Functions
}

\author{
By Yudell L. Luke and Richard L. Coleman
}

Abstract. In a previous paper [1] one of us developed an expansion for the confluent hypergeometric function in series of Bessel functions. A different expansion of the same kind given by Buchholz [2] was also studied. Since publication of [1], it was found that Rice [3] has also developed an expansion of this type, and yet a fourth expansion of this kind can be deduced from some recent work by Alavi and Wells [4]. In this note, we first deduce a multiplication formula for the Gaussian hypergeometric function which generalizes a statement of Chaundy, see (11), page 187 of [5], and includes a multiplication theorem for the confluent hypergeometric functions due to Erdélyi, see (7), page 283 of [5]. Our principal result is specialized to give an expansion of the confluent hypergeometric function in series of Bessel functions which includes the four above as special cases. With the aid of the Laplace transform, the latter result is used to derive an expansion of the Gaussian hypergeometric function in series of functions of the same kind with changed argument. This is advantageous since, throughout most of the unit disc, the change in argument leads to more rapidly converging series. For special values of the parameters, the expansion degenerates into known quadratic transformations.

1. A Multiplication Theorem for the Gaussian Hypergeometric Function. We first prove

$$
\begin{array}{r}
{ }_{2} F_{1}\left(\begin{array}{c}
a, b \\
c
\end{array} \mid \lambda z\right)=\sum_{k=0}^{\infty} \frac{(-)^{k}(\alpha)_{k}(\beta)_{k} z^{k}}{k !(\gamma+k)_{k}}{ }_{4} F_{3}\left(\begin{array}{c}
-k, k+\gamma, a, b \mid \lambda) \\
\alpha, \beta, c
\end{array} \mid \lambda\right) \\
\quad \times{ }_{2} F_{1}\left(\begin{array}{c}
\alpha+k, \beta+k \\
\gamma+1+2 k
\end{array} \mid z\right) .
\end{array}
$$

For notation and definitions, see Erdélyi [5], Chapters 2 and 4 . Note that the parameters $\alpha, \beta$ and $\gamma$ are free, provided that the resulting expression is meaningful. Let $q_{m}$ be the coefficient of $\lambda^{m}$ on the right-hand side of (1.1). Then

$$
\begin{gathered}
q_{m}=\sum_{k=n}^{\infty} \frac{(-)^{k} z^{k}(\alpha)_{k}(\beta)_{k}(-k)_{m}(\gamma+k)_{m}(a)_{m}(b)_{m}}{k !(\gamma+k)_{k}(\alpha)_{m}(\beta)_{m}(c)_{m} m !}\left(\begin{array}{c}
\alpha+k, \beta+k \\
\gamma+1+2 k
\end{array} \mid z\right) \\
=\frac{(a)_{m}(b)_{m} z^{m}}{(c)_{m} m !} \sum_{n=0}^{\infty} \frac{(\alpha+m)_{n}(\beta+m)_{n} z^{n}}{n !} \sum_{k=0}^{\infty} \frac{(-)^{k} \Gamma(\gamma+2 m+k)}{k ! \Gamma(\gamma+2 m+2 k)} \\
\times \frac{\Gamma(\gamma+1+2 m+2 k) \Gamma(n+1)}{\Gamma(\gamma+1+2 m+n+k) \Gamma(n+1-k)} \\
=\frac{(a)_{m}(b)_{m} z^{m}}{(c)_{m} m !} \sum_{n=0}^{\infty} \frac{(\alpha+m)_{n}(\beta+m)_{n} z^{n}}{n !(\gamma+1+2 m)_{n}} B_{n}
\end{gathered}
$$

Received September 30, 1960. This research was supported jointly by the United States Air Force through the Air Force Office of Scientific Research of the Air Research and Development Command and by the United States Navy through the Applied Mathematics Laboratory of the David W. Taylor Model Basin. 
where

$$
\begin{aligned}
B_{n}={ }_{2} F_{1} & \left(\begin{array}{c|c}
-n, \gamma+2 m \\
\gamma+1+2 m+n
\end{array} \mid 1\right) \\
& -\frac{2 n}{\gamma+1+2 m+n}{ }_{2} F_{1}\left(\begin{array}{c}
-n+1, \gamma+1+2 m \\
\gamma+2+2 m+n
\end{array}\right)
\end{aligned}
$$

Now using the fact, see (46), page 104 of [5],

$$
\begin{aligned}
&{ }_{2} F_{1}\left(\begin{array}{c}
A, B \\
C
\end{array} \mid 1\right)=\frac{\Gamma(C) \Gamma(C-A-B)}{\Gamma(C-A) \Gamma(C-B)}, \\
& C \neq 0,-1,-2, \cdots, \quad R(C-A-B)>0,
\end{aligned}
$$

we see that

$$
B_{n}=\left\{\begin{array}{lll}
1 & \text { if } & n=0 \\
0 & \text { if } & n>0
\end{array}\right.
$$

Thus (1.2) becomes

$$
q_{m}=\frac{(a)_{m}(b)_{m} z^{m}}{(c)_{m} m !}
$$

which proves (1.1). Chaundy's statement is (1.1) with $\lambda=1$.

In (1.1), set $b=\beta$, replace $z$ by $z / b$ and let $b \rightarrow \infty$. Then

$$
\begin{aligned}
\Phi(a, c ; \lambda z)=\sum_{k=0}^{\infty} \frac{(-)^{k}(\alpha)_{k} z^{k}}{k !(\gamma+k)_{k}} \\
\cdot{ }_{3} F_{2}\left(\begin{array}{c}
k, k+\gamma, a \\
\alpha, c
\end{array} \mid \lambda\right) \Phi(\alpha+k, \gamma+1+2 k ; z) .
\end{aligned}
$$

Erdélyi's expansion theorem is (1.7) with $\alpha=a$. Again in (1.1), put $b=c$, replace $\lambda$ by $\lambda / a$ and let $a \rightarrow \infty$. Then

$$
e^{\lambda z}=\sum_{k=0}^{\infty} \frac{(-)^{k}(\alpha)_{k}(\beta)_{k} z^{k}}{k !(\gamma+k)_{k}}{ }_{2} F_{2}\left(\begin{array}{c}
-k, k+\gamma \\
\alpha, \beta
\end{array} \mid \lambda\right){ }_{2} F_{1}\left(\begin{array}{c}
\alpha+k, \beta+k \\
\gamma+1+2 k
\end{array} \mid z\right)
$$

It can be noted that expansion formulas of the type (1.1) for the $G$-function (a generalization of hypergeometric functions) have been studied in a series of papers by Meijer [6]. However, neither (1.1) nor (1.7)-(1.8) can be deduced from his work.

2. Expansion of the Confluent Hypergeometric Function in Series of Bessel Functions. In (1.7), put $\gamma+1=2 \alpha$ and note that

$$
\Phi(\alpha+k ; 2 \alpha+2 k ; z)=\Gamma\left(\alpha+k+\frac{1}{2}\right)(4 / z)^{\alpha+k-1 / 2} e^{z / 2} I_{\alpha+k-1 / 2}(z / 2) .
$$

Then

$$
\begin{aligned}
\Phi(a, c ; \lambda z)= & \frac{e^{z / 2} \Gamma(2 \delta+1)}{\left(\frac{1}{2}\right)_{\delta} z^{\delta}} I_{\delta}(z / 2) \\
& +\frac{2 e^{z / 2}}{\left(\frac{1}{2}\right)_{\delta} z^{\delta}} \sum_{k=1}^{\infty} \frac{(-)^{k}(\delta+k) \Gamma(2 \delta+k)}{k !} R_{k}(a, c, \delta ; \lambda) I_{k+\delta}(z / 2)
\end{aligned}
$$


where $\alpha-\frac{1}{2}=\delta$ and

$$
R_{k}(a, c, \delta ; \lambda)={ }_{3} F_{2}\left(\begin{array}{c}
-k, k+2 \delta, a \\
\frac{1}{2}+\delta, c
\end{array} \mid \lambda\right) .
$$

Assume $\lambda=1$ and write $R_{k}(a, c, \delta, 1)=R_{k}(a, c, \delta)$. If $\delta=0$, we get the result in [1], and $\delta=a-\frac{1}{2}$ yields the Buchholz expansion. Another proof of the latter has been given by Slater [7]. $\delta=\frac{1}{2}$ and $\delta=c-\frac{1}{2}$ give the expansions in [3] and [4], respectively. The ${ }_{3} F_{2}$ given in [4] is not of the form (2.3), but may be reduced to it using known transformation properties of ${ }_{3} F_{2}$ 's (see Bailey [8]).

Properties of the $R_{k}$ 's for $\lambda=1$ are next of interest. These are recorded without proof as the argument is much akin to that developed in [1] for the case $\delta=0$.

$$
\begin{gathered}
R_{k}(a, c, \delta)=(-)^{k} R_{k}(c-a, c, \delta) ; R_{k}(a, a, \delta)=(-)^{k} \\
(k+c)(k+2 \delta) R_{k+1}(a, c, \delta) \\
=2(c-2 a)(k+\delta) R_{k}(a, c, \delta)+k(k+2 \delta-c) R_{k-1}(a, c, \delta) .^{*} \\
R_{k}(a, 2 a, \delta)=0, \quad k \text { odd } ; \quad R_{2 k}(a, 2 a, \delta)=\frac{\left(\frac{1}{2}\right)_{k}\left(\delta-a+\frac{1}{2}\right)_{k}}{\left(\frac{1}{2}+a\right)_{k}\left(\frac{1}{2}+\delta\right)_{k}} \\
R_{k}\left(a, c, c-a-\frac{1}{2}\right)=\frac{(c-2 a)_{k}}{(c)_{k}} \\
R_{k}\left(a, c, a-\frac{1}{2}\right)=\frac{(-)^{k}(2 a-c)_{k}}{(c)_{k}}
\end{gathered}
$$

If $\delta=\frac{1}{2}$, then

$$
\operatorname{Erf} z=\int_{0}^{z} e^{-t^{2}} d t=e^{-z^{2} / 2} \pi^{1 / 2} \sum_{k=0}^{\infty} I_{k+1 / 2}\left(z^{2} / 2\right)
$$

The convergence of $(2.9)$ is inferior to that of (2.7) of [1].

3. Expansion of the Gaussian Hypergeometric Function in Series of Functions of the Same Kind with Changed Argument. Now combine the known Laplace transforms [9]

$$
\begin{gathered}
\int_{0}^{\infty} e^{-p t} t^{b-1} I_{\nu}(t) d t=\frac{\Gamma(\nu+b)(p-q)^{\nu}}{\Gamma(\nu+1) q^{b}}{ }_{2} F_{1}\left(1-b, b ; \nu+1 ; \frac{q-p}{2 q}\right), \\
q=\left(p^{2}-1\right)^{1 / 2}, \quad R(b)>0, \quad R(p)>1, \\
\int_{0}^{\infty} e^{-(p+1) t} t^{b-1} \Phi(a ; c ; 2 t) d t=\frac{\Gamma(b)}{(p+1)^{b}}{ }_{2} F_{1}\left(a, b ; c ; \frac{2}{p+1}\right), \\
R(b)>0, \quad R(p)>0
\end{gathered}
$$

* A proof after the manner of (2.5) shows that $R_{k}(a, c, \delta ; \lambda)$ satisfies a five-term recurrence formula. The expression does not seem to be of general interest and so is omitted. 
with (2.2) for $\lambda=1$ and put $z(p+1)=2$. Then for $|z|<1$,

$$
\begin{array}{r}
{ }_{2} F_{1}(a, b ; c ; z)=\frac{w^{\delta-b} 2^{2 \delta}}{(1+w)^{2 \delta}} \sum_{k=0}^{\infty} \frac{(-)^{k}(b)_{k}(2 \delta)_{k}}{k !(\delta)_{k}} R_{k}(a, c, \delta)\left(\frac{1-w}{1+w}\right)^{k} \\
\quad \times{ }_{2} F_{1}\left(1-b+\delta, b-\delta ; k+\delta+1 ;-\frac{(1-w)^{2}}{4 w}\right),
\end{array}
$$

where $w=(1-z)^{1 / 2}$ is real and positive if $z$ is real and $0 \leqq z<1$. Equation (3.3) is convenient since the change in argument leads to more rapidly convergent series throughout most of the unit disc. To see this, note that $|z| \geqq\left|\frac{1-w}{1+w}\right|$ for all $|z| \leqq 1$ and equality holds only for $z=0$ and $z=1$. Also, if $z$ is real, $|z| \geqq$ $(1-w)^{2} / 4 w=v$ whenever $z \leqq \frac{5(41)^{1 / 2}-1}{32}=0.96924$. If $z=\rho e^{i \theta}$, then by numerical computation, we find that $|z| \geqq|v|$ holds for $\rho=0.97,0.98,0.99$ and 1.0 if $\theta \geqq 0.0074,0.0260,0.0331$ and 0.0365 , respectively. Another advantage is that $\delta$ is a free parameter. Thus, if $\delta=b$ or $\delta=b-1$, the ${ }_{2} F_{1}$ on the right is unity. If also the parameters $a, b$ and $c$ are such that $R_{k}$ is a product of gamma functions as in (2.6)-(2.8), then the right-hand side of (3.3) is a hypergeometric function and we can recover known quadratic transformations. For example, if $\delta=b$, and $c=2 a$, then

$$
{ }_{2} F_{1}(a, b ; 2 a ; z)=\left(\frac{2}{1+w}\right)^{2 b}{ }_{2} F_{1}\left(b-a+\frac{1}{2}, b ; \frac{1}{2}+a ;\left(\frac{1-w}{1+w}\right)^{2}\right)
$$

and from the latter, one can readily deduce the Gauss transformation for the complete elliptic integral of the first kind.

If $a=\frac{1}{2}, b=1, c=\frac{3}{2}$ and $\delta=0$, (3.3) yields expansions for $\arctan a^{-1}$ and $\log (1+a)$ which are special cases of Chebyshev expansions previously given by Luke [10].

We now obtain a useful expansion for the complete elliptic integral of the first kind $K(k)$ when the modulus is near unity. It is known that

$$
\begin{aligned}
K(k)+\frac{2}{\pi}\left(\log k^{\prime}\right) K\left(k^{\prime}\right) & =\sum_{m=0}^{\infty}\left\{\frac{\left(\frac{1}{2}\right)_{m}}{m !}\right\}^{2} \\
\cdot & \left\{\psi(m+1)-\psi\left(m+\frac{1}{2}\right)\right\}\left(k^{\prime}\right)^{2 m},\left(k^{\prime}\right)^{2}=1-k^{2},
\end{aligned}
$$

which is the same as

$$
-\frac{\partial}{\partial a}\left[{ }_{2} F_{1}\left(a-\frac{1}{2}, \frac{1}{2} ; a ;\left(k^{\prime}\right)^{2}\right)\right]_{a=1}+\frac{4}{\pi}(\log 2) K\left(k^{\prime}\right) .
$$

To evaluate the partial derivative, use (3.3) with $\delta=0$ and $b=\frac{1}{2}$ where first $a$ is replaced by $a-\frac{1}{2}$ and then $c$ is replaced by $a$. It follows that

$$
\frac{\partial}{\partial a}\left\{R_{k}\left(a-\frac{1}{2}, a, 0\right)\right\}_{a=1}= \begin{cases}-\frac{1}{k}, \quad k>0 \\ 0, \quad k=0\end{cases}
$$


and so

$$
\begin{aligned}
K(k)=\frac{2}{\pi}\left(\log \frac{4}{k^{\prime}}\right) K\left(k^{\prime}\right)+2 k^{-1 / 2} \sum_{m=1}^{\infty} & \frac{(-)^{m}\left(\frac{1}{2}\right)_{m}}{m ! m}\left(\frac{1-k}{1+k}\right)^{m} \\
& \times{ }_{2} F_{1}\left(\frac{1}{2}, \frac{1}{2} ; m+1 ;-\frac{(1-k)^{2}}{4 k}\right) .
\end{aligned}
$$

Midwest Research Institute

Kansas City, Missouri

1. Y. L. LUKE, "Expansion of the confluent hypergeometric function in series of Bessel functions," $M T A C$, v. 13, Oct. 1959, p. 261-271. (The following errata have been noted in this reference. In (1.17) remove $z$ in $\frac{a z}{c}$; in (5.4) the coefficient of $A_{k-2}$ should read $a h(h-1)$; in (6.9) for $z^{k}$ read $(z / 2)^{k}$.)

2. H. BuCHHOLz, Die Konfluente Hypergeometrische Funktionen, Springer, 1953, p. 130.

3. S. O. RICE, "Some properties of ${ }_{3} F_{2}$...", Duke Math. J., v. 6, March 1940, p. 108-119.

4. Y. Alavi, \& C. P. Wells, "Expansions of parabolic wave functions," Proc. Amer. Math. Soc., v. 10, Dec. 1959, p. 876-880.

5. A. Erdfuxi, et al., Higher Transcendental Functions, v. 1, McGraw-Hill Book Company, Inc., 1953. 14-18.

6. C. S. MeIJer, "Expansion theorems for the G-function," Indag. Math., (1952-1956), p.

7. L. Slater, "Expansions of generalized Whittaker functions," Proc. Cambridge Philos. Soc., v. 50, 1954, p. 628-631.

8. W. N. BaILEY, Generalized Hypergeometric Series, Cambridge University Press, 1935.

9. A. ERDfiri, et al., Tables of Integral Transforms, v. 1, McGraw-Hill Book Company, Inc., 1954, p. 196 and p. 215.

10. Y. L. LUKE, "On the computation of $\log z$ and $\operatorname{arc} \tan z$," MTAC, v. 11, Jan. 1957, p. 16-18. 